Extract of rabbit muscles was used for the investigation. To the muscle extract, buffered at a $p H$ of about $7 \cdot 5$, sodium fluoride phloridzin and finally the substrate hexosediphosphate were added. In the samples the decrease of P-compounds labile to acid hydrolysis and the increase of $\mathrm{P}$-compounds resistant to acid hydrolysis were determined. Further, the hydrolysis of P-compounds in alkali at room temperature before and after oxidation with iodine was determined in order to estimate the accumulation of dihydroxyacetonphosphate and glyceraldehydphosphat $\Theta^{3,4}$. In order to study the action of phloridzin on the formation of the last two substances, it is necessary to dilute the extract more than 100 times $^{3}$.

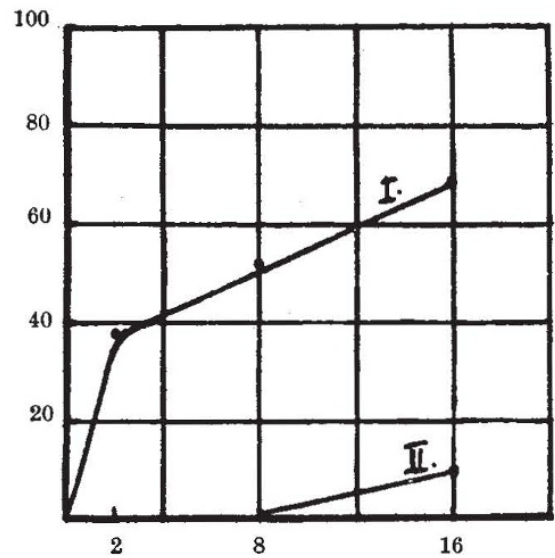

Frg. 1. Relation of percentage conversion into acid-resistant P-compounds (ordinates) to time in minutes (abscissa). I : without phloridzin ; II : with $N / 100$ phloridzin.

The velocity of the conversion of hexosediphosphate into dihydroxyacetonphosphate and of this into glyceraldehydphosphate is not affected by phloridzin in concentrations of about $N / 100$. On the other hand, the dismutative conversion of triosephosphate into phosphoglyceric acid and glycerophosphoric acid is inhibited 70-100 per cent by phloridzin in the same concentrations. In the same extract with starch as substrate, the well-known inhibition of the phosphorylation was observed. At the phloridzin concentration mentioned above, this inhibition was of the same order as the inhibition of the dismutation.

Further experiments must determine whether the inhibition of oxidation-reduction is an essential action of phloridzin.

University Institute of

H. KALCKAR.

Medical Physiology,

Copenhagen.

${ }^{1}$ Lundsgaard, Biochem. Z., 264, 209; 1932.

2 Barth, Biochem. Z., 270, 63; 1934 .

Meyerhof and Iohmann, Biochem. Z., 271, 89 ; 1934

- Meyerhof and Kiessling, Biochem. Z., 279, 40 ; 1935.

\section{A Toxic Emanation from Rubber}

Apparatus of several types for measuring and controlling the humidity of the air in entomological experiments was recently described by us ${ }^{1}$. We mentioned a convenient type of glass jar, which is now in rather common use among entomologists and botanists, for studying the effects of climatic factors on insects or plants. It is a commercial glass storage jar with a screw-on metal cap. The joint between the glass and metal is sealed by a thick rubber washer. The humidity inside the jar is controlled by means of a layer of a suitable solution (of potash, acid, etc.) and the insects are placed in the jar in a celluloid and gauze container.

Since describing this apparatus, and after using it frequently, one of us has discovered a potential source of error. If new rubber washers are used to seal the jars, they may, under certain conditions only, give out a toxic substance. This poisonous emanation is much more powerful when the humidity in the jar is high than when it is low. Thus sometimes moist air may*appear to be more unfavourable to an insect than dry, when the difference is actually due to a fault of the apparatus. Certain conclusions obtained using this type of apparatus, for example with regard to the unfavourable effects of high humidities on tsetse $\mathrm{fly}^{2}$, must therefore be accepted with caution.

After exposure to air for four months, the rubber washers which we have been using completely lose their toxic properties, and it is safe to use them even in saturated air. Different samples of rubber vary considerably, and any apparatus for investigating effects of humidity which contains rubber joints should be carefully tested.

K. Mellanby.

P. A. Buxton.

1 Buxton and Mellanby, Bull. Ent. Re8., 25, 171; 1934.
2 Buxton and Lewis, Phil. Trans. Roy. Soc., B, 224, 175; 1934.

\section{Cytological Significance of the Nature of Sexual Fusion in Hymenomycetes}

The late Hans Kniep from 1915 onwards and Mlle. Bensaude in 1918 described the union of two mycelia of + and - strains as a necessary preliminary to the formation of the sporophore in heterothallic species of Hymenomycetes. In such cases, plasmogamy is usually separated from karyogamy in the basidium by several successive divisions of conjugate nuclei through clamp connexions. This is held as indicating a "degeneration of the sexual process", and as the principal point of distinction from the type of sexual fusion known in green plants, where there is simultaneous fusion of protoplasm and nuclei of two conjugating gametes. But a closer reflection would lessen this difference.

No doubt in the higher plants we have fusion of the two nuclei with the formation of the zygote and the initiation of the diplophase, but not the fusion of two sets of chromosomes (male and female), which simply lie together in the fusion nucleus; the actual fusion of chromosomes is delayed there by almost the same long steps until the prophase of the reduction division or meiosis in connexion with micro. sporogenesis or megasporogenesis. In the Hymenomycetes, on the other hand, the fusion of the two nuclei in the basidium is followed almost at once by the fusion of their chromosomes in connexion with meiosis. Thus, the only difference is that in the Hymenomycetes the two conjugate nuclei from two different strains (plus and minus) are not in actual contact with each other in the form of one fusionnucleus as in the higher plants, though they lie very close together in each cell of the hyphæ.

Botanical Laboratory, S. R. BOSE.

Carmichael Medical College,

Calcutta. Oct. 28. 